\title{
Desenvolvimento de uma interface gráfica educacional para ensino de elementos finitos aplicado a problemas de viga sob base elástica
}

\author{
Neves, N. S ${ }^{1 *}$; Pinheiro, V. P. ${ }^{2}$; Camargo, R. S. ${ }^{1}$; \\ 1 Programa de Pós-Graduação em Engenharia Civil, Universidade Federal do Espírito Santo, Vitória, ES, Brasil. \\ 2 Programa de Pós-Graduação em Engenharia Mecânica, Universidade Federal do Espírito Santo, Vitória, ES, Brasil.
}

* e-mail: natan.sian@gmail.com

\section{Resumo}

A seara do ensino em engenharia é uma vertente que movimenta pesquisa científica e apresentam inúmeros desafios, exigindo a otimização na relação docente-discente. Desta maneira, o presente artigo busca apresentar uma plataforma gráfica didática para auxiliar no processo de ensino-aprendizagem de elementos finitos e no estudo do comportamento de estruturas reticulares de dois graus de liberdade. Para tanto, usa-se os recursos gráficos do Matlab para criação do modelo iterativo computacional e aplicam-se os procedimentos de elementos finitos de Galerkin para a solução da equação diferencial de uma viga de Euler-Bernoulli com base elástica de Winkler. A validação é conduzida por soluções analíticas e resultados numéricos da literatura. Em síntese, o programa desenvolvido apresentou bons resultados quanto à predição do campo de deslocamentos e de esforços internos, mostrando-se também como uma ferramenta complementar no embasamento teórico dos discentes.

Palavras chaves: Elementos Finitos, Euler-Bernoulli, Didática; Ensino.

\begin{abstract}
The field of engineering education is a strand that moves scientific research and presents numerous challenges, requiring optimization in the teacher-student relationship. In this way, the present article seeks to present a didactic graphic platform to assist in the teaching-learning process of finite elements and in the study of the behavior of reticular structures of two degrees of freedom. To do so, we use Matlab's graphical features to create the computational iterative model and apply Galerkin's finite element procedures to the solution of the differential equation of an Euler-Bernoulli beam with Winkler elastic base. The validation is driven by analytical solutions and numerical results of the literature. In summary, the developed program presented good results regarding the prediction of the field of displacements and of internal efforts, also being a complementary tool in the theoretical foundation of the students.
\end{abstract}

Keywords: Finite Elements, Euler-Bernoulli, Didactics; Teaching.

\section{Introdução}

A análise estrutural é uma área que abrange inúmeras aplicações de suma importância para indústria. Tal demanda requer uma base sofisticada de conceitos físicos e modelagem matemática contínua e discreta. Neste cenário, a transmissão de conhecimento não é uma tarefa trivial e transporta diversos desafios.

Neste contexto, com os avanços tecnológicos, houve um crescente desenvolvimento de programas comerciais que buscam solucionar problemas estruturais com distintos graus de complexidade. Em contrapartida, destinado a complementar o processo de ensino, também houve desenvolvimento de diversos programas livres como, por exemplo, Ftool, INSANE, LESM e MDSolids. Contudo, existe ainda uma carência de programas e interfaces pedagógicas voltadas ao aprendizado dos discentes, mesmo tendo em vista que o desenvolvimento destas ferramentas auxiliares reforçam o entendimento e intensificam o interesse dos discentes nas disciplinas [1]. 


\section{Modelagem Numérica}

A equação de governo do problema de viga em base elástica baseia-se no equilíbrio de um elemento diferencial sujeito a uma carga transversal $q(x)$ e uma reação proporcional ao deslocamento, desta maneira, pode-se escrever a Equação (1).

$$
E I \frac{d^{4} w}{d x^{4}}+k_{v} w=q(x)
$$

Em que, $E$ é o modulo de elasticidade, $I$ momento de inércia, $w$ deslocamento e $k_{v}$ é o coeficiente de reação vertical. Desta forma, tendo posto o modelo diferencial do problema, a formulação de elementos finitos de Galerkin inicia-se escrevendo a Equação (1) como uma sentença integral associada a uma função de forma $N_{i}$. Aplicando a regra da cadeia e o teorema da divergência, após algumas manipulações [2], chegar na Equação (2).

$$
\begin{aligned}
& \int \frac{d^{2} N_{i}}{d x^{2}} E I \frac{d^{2} w}{d x^{2}} d \Omega+\int k_{v} w N_{i} d \Omega= \\
& -E I\left[\frac{d^{3} w}{d x^{3}} N_{i}-\frac{d^{2} w}{d x^{2}} \frac{d N_{i}}{d x}\right]_{x_{i}}^{x_{f}}+\int q N_{i} d \Omega
\end{aligned}
$$

A forma fraca do problema não apresenta nenhuma aproximação, então, introduzindo as funções de interpolação com base nos polinômios de Hermite [2], pode-se construir a solução aproximada da função deslocamento, conforme Equação (3).

$$
\begin{aligned}
& w=\sum \bar{w}_{j} N_{j}= \\
& \bar{w}_{1} N_{1}+\bar{w}_{2} N_{2}+\bar{w}_{3} N_{3}+\bar{w}_{4} N_{4}
\end{aligned}
$$

Desta forma, aplica-se a Equação (3) na Equação (2), assim é possível obter o sistema algébrico final, conforme posto na Equação (4).

$$
\begin{aligned}
& {[\mathbf{K}]\{w\}=\{\mathbf{F}\}} \\
& \mathbf{K}=\int \frac{d^{2} N_{i}}{d x^{2}} E I \frac{d^{2} N_{j}}{d x^{2}} d \Omega+\int N_{j} k_{v} N_{i} d \Omega \\
& \mathbf{F}=Q_{i}+\int q N_{i} d \Omega
\end{aligned}
$$

Sendo que $Q_{i}$ representa o vetor de esforço nas extremidades das barras.

\section{Programa Computacional}

O programa desenvolvido é baseado na técnica de elementos finitos, sendo codificada em Matlab e estruturado basicamente por três etapas globais, conforme Figura 1. Primeiramente, realiza-se o pré-processamento, onde ocorre a entrada de dados iniciais por meio da leitura de arquivo de texto contendo propriedades físicas e geométricas dos elementos, carregamentos externos, coordenadas, conectividade, restrições dos nós e o coeficiente da base elástica.

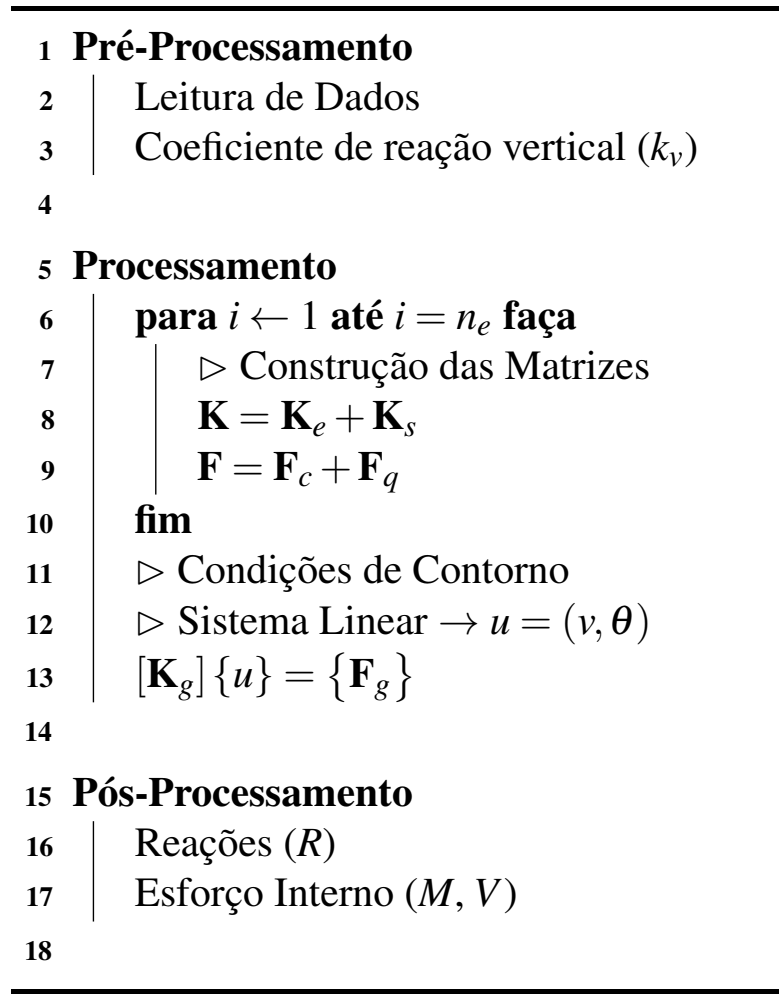

Figura 1: Lógica global da implementação do MEF

Em seguida, o processamento inicia-se com a montagem da matriz de rigidez $\mathbf{K}$ e do vetor de carga $\mathbf{F}$, imposição das restrições em cada nó e por fim a solução do sistema linear global. Desta forma, após obter o vetor resposta com deslocamentos verticais e rotações, realiza-se o pós-processamento, onde determina-se as reações de apoio e os esforços internos em cada barra.

Para validar o algoritmo desenvolvido, considere inicialmente, a viga biapoiada com uma carga concentra no meio vão, conforme ilustra a Figura 1. 


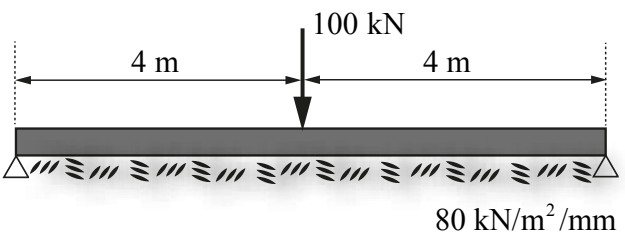

Figura 2: Viga biapoiada com carga concentrada

A seção transversal é retangular de 300x $500 \mathrm{~mm}$ e a solução exata pode ser obtida facilmente na literatura [3].

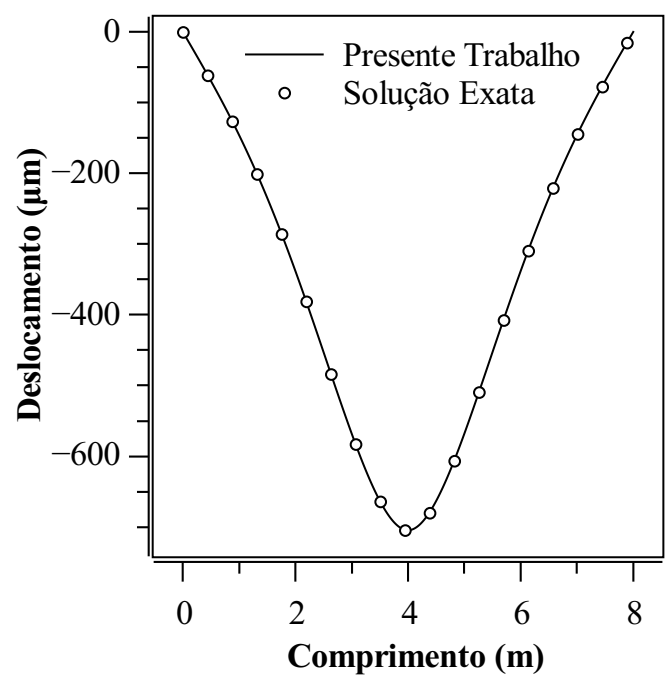

Figura 3: Campo de deslocamento da viga

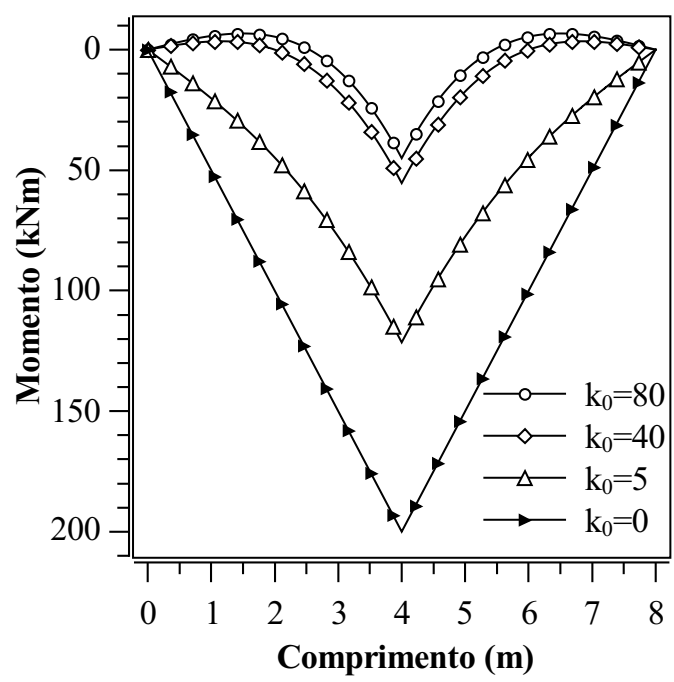

Figura 4: Comportamento do Momento fletor

A Figura 3 apresenta a comparação de resultados para o deslocamento vertical. Observe que o comportamento apresentado entre as curvas são bem ajustados. Em adicional a este caso, vale realizar uma análise limítrofe. Para tanto, a Figura 4 expõe uma análise paramétrica em relação a variação do coeficiente de reação. Note que quanto maior a rigidez do solo, menos pronunciado é o efeito de flexão da viga. Vale destacar ainda, que quando não existe base elástica $\left(k_{v}=0\right)$, a solução recai para resposta clássica de flexão, atingindo o momento máximo de $200 \mathrm{kNm}$.

O segundo caso é uma viga de seção transversal 500x250mm com borda livre e apoiada em uma base elástica sujeita a carga distribuída, momentos e forças concentradas [4], como ilustra a Figura 5.

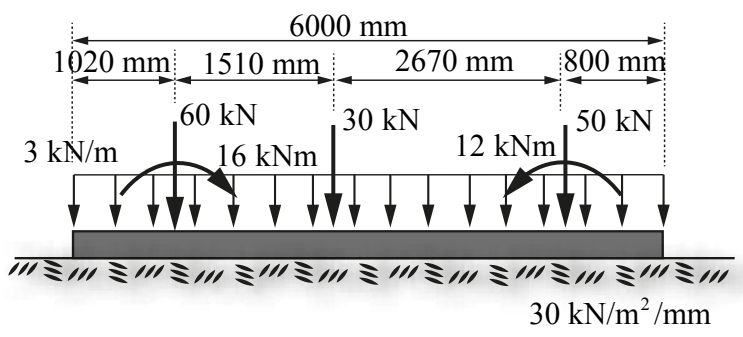

Figura 5: Dimensões e carregamentos na viga

Para quantificar os resultados, a Tabela 1 apresenta o resultado do esforço cortante e momento fletor para os pontos medidos a partir da borda livre esquerda na cota $1210 \mathrm{~mm}$, $2530 \mathrm{~mm}$ e $5200 \mathrm{~mm}$, ou seja, os pontos de aplicação das cargas concentradas na viga.

Tabela 1: Comparação de resultados com a literatura

\begin{tabular}{cllll}
\hline \multirow{2}{*}{$\begin{array}{c}\text { Dist. } \\
(\mathrm{m})\end{array}$} & \multicolumn{2}{c}{$\begin{array}{c}\text { Cortante } \\
\text { Máximo }(\mathrm{kN})\end{array}$} & \multicolumn{2}{c}{ Momento } \\
\cline { 2 - 5 } & MÉximo & $\begin{array}{l}\text { Glyn J. } \\
(1997)\end{array}$ & MEF & \multicolumn{2}{c}{$\begin{array}{l}\text { Glyn J. } \\
(1997)\end{array}$} \\
\hline 1,02 & $-37,87$ & $-37,92$ & $+25,83$ & $+25,83$ \\
2,53 & $-22,70$ & $-22,77$ & $+3,58$ & $+3,58$ \\
5,20 & $+33,52$ & $+33,59$ & $+18,31$ & $+18,31$ \\
\hline
\end{tabular}

Perceba que todos os resultados de momento fletor apresentam boa concordância com a solução de referência, todavia, a pequena variação que existe no esforço cortante é devido a derivada de terceira ordem da solução aproximação ser constante em cada elemento. Tendo posto a validação, é interessante, ao nível de ensino de engenharia, desenvolver uma interface gráfica educacional com as bases de cálculos apresentadas anteriormente. Então, a construção de tal ferramenta é elaborada em ambiente que permite ao usuário uma interação direta com o programa via elementos gráficos (botões, slider, menus e etc). Sendo que a dinâmica do programa é realizada 


\section{Blucher Proceedings

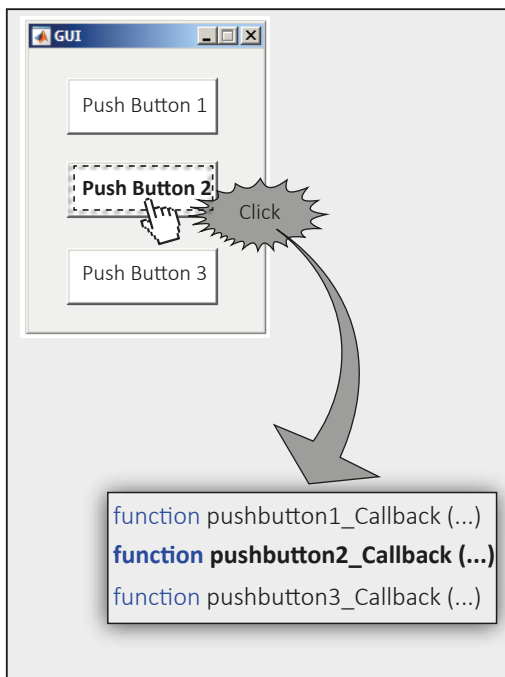

(a)

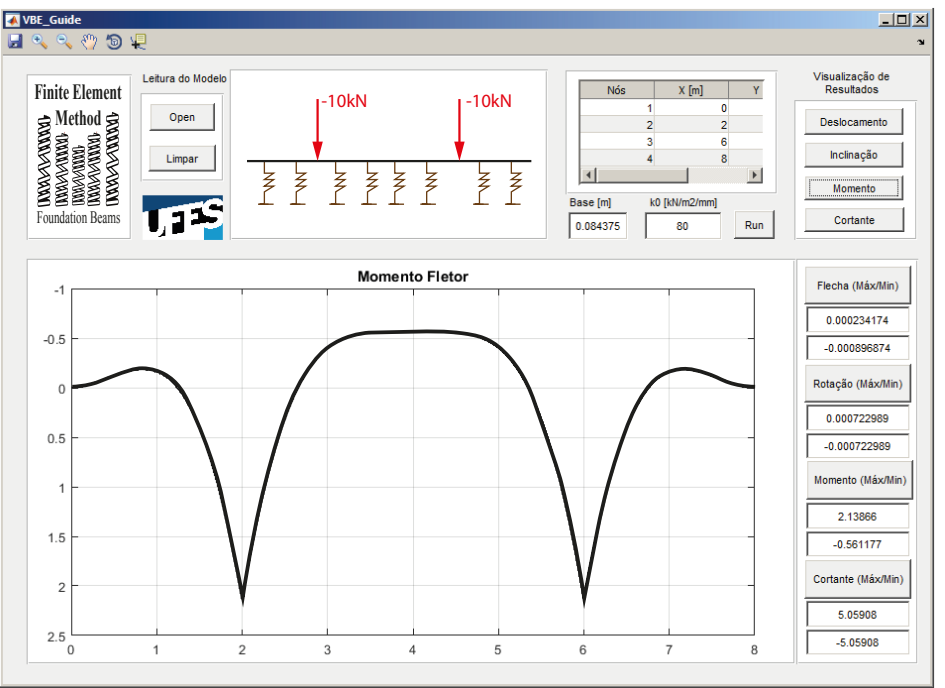

(b)

Figura 6: (a) Ideia da Programação Orientada à Eventos; (b)Interface gráfica do programa desenvolvido

por eventos, isto é, as diferentes ações provenientes do usuário sobre elementos gráficos da interface. Cada evento esta interligado por uma função denotada Callback [5], como mostra o esquema da Figura $6 a$.

Em relação ao problema tratado físico, a interface do programa é ilustrada na Figura 6b. Os procedimentos sequenciais são basicamente parelhos com os apresentados no pseudocódigo da Figura 1. Os resultados são apresentados por meio das curvas de deslocamentos, rotação, momento fletor e esforço cortante.

Com tal ferramenta, os discentes podem realizar análises limítrofes em relação a influência na resposta para diferentes valores do coeficiente de rigidez do base elástica. Além disso, possibilita ao usuário uma investigação acerca dos valores máximos das variáveis de interesse, sendo um recurso importante para determinar os esforços solicitantes de cálculo ou deslocamento máximos em dimensionamentos de vigas de fundação.

\section{Considerações Finais}

O programa desenvolvido para análise do comportamento de vigas sob base elástica apresentou resultados satisfatórios em relação aos soluções de referência. Em adicional, com uma interface simples e didática é possível ex- plorar rapidamente o comportamento de vigas com ou sem reação da base elástica, permitindo aos discentes uma ferramenta potencializadora para auxiliar na compreensão do comportamento físico básico de estruturas e a inserção de conceitos primários e da lógica do método de elementos finitos.

\section{Referências}

[1] MOREIRA, R. N.; Roque, L. S. P. Sistema gráfico interativo para ensino de análise estrutural através do método dos elementos finitos. Revista de Ensino de Engenharia, v. 37, n. 1, p. 76-87, 2018.

[2] REDDY, J. N. An introduction to the finite element method. New York: McGraw-hill, 1993. v. 2

[3] DEN HARTOG, J. P. Advanced strength of materials. New York: McGraw-Hill. 1952.

[4] JONES, G. Analysis of beams on elastic foundations: using finite difference theory. Thomas Telford, 1997.

[5] RANGEL, R. L.; MARTHA, L.F. LESM-An object-oriented MATLAB program for structural analysis of linear element models. Computer Applications in Engineering Education. 2018 